\title{
Maintaining visibility constraints during tele-echography with ultrasound visual servoing
}

\author{
Tao Li, Olivier Kermorgant and Alexandre Krupa
}

\begin{abstract}
This paper presents a multi-task control method to maintain the visibility of an anatomic element of interest while the doctor tele-operates a $2 \mathrm{D}$ ultrasound probe held by a medical robot. The prior task consists in automatically maintaining several visual constraints that guarantee an intersection between the ultrasound image plane and the anatomic object of interest and the second task allows the medical expert to manually apply probe motion through tele-operation. Unlike classical visual servoing technique which continually regulate the current visual features to desired values, our control approach gradually activates the regulation of one or several ultrasound visual features that go close to fixed limits in such a way to keep them in a safe domain. The main advantage of this approach is to give to the clinician the control of all the degrees of freedom of the probe to examine the patient while automatically preserving the visibility of the element of interest if required. Both simulations and experiments performed on an abdominal phantom demonstrate the efficiency of the visibility assistance task.
\end{abstract}

Index Terms - Tele-echography, visual servoing, visibility constraints, redundancy

\section{INTRODUCTION}

Among the different medical imaging modalities, such as ultrasound (US) imaging, X-ray imaging, magnetic resonance imaging (MRI) or Computerized Tomography (CT), the US imaging has many advantages on one hand for the doctors and on the other hand for the patients. Indeed the US modality has three major assets, it is cheap, real-time and noinvasive for the human body. Moreover the US transducer is portable and US imaging can be performed even if ferromagnetic instruments are present in the observed image. Based on these reasons, tele-operable portable ultrasound robotic system has been widely used by radiologists to perform remote ultrasound examinations of patients.

In the 90's, the tele-ultrasound examinations [1] required an ultrasound technician to be present by the side of the patient to manually manipulate the US probe under the order of an expert thanks to the use of a video conferencing equipment system. After that, the evolution was to actuate the US probe by a robot in such a way to automatically follow a pre-specified trajectory [2]. However, the necessity to planify a probe trajectory reduces the flexibility of this examination procedure.

In the last decade, several research teams developed robotic systems for tele-echography as for example in [3], [4] and [5]. With such systems, a remote radiologist can teleoperate an ultrasound probe held by a robot in such a way

Tao Li, Olivier Kermorgant and Alexandre Krupa are with INRIA Rennes Bretagne Atlantique - IRISA, Campus de Beaulieu, 35042 Rennes, France. E-mail: Firstname.Namedinria.fr it follows the movement applyed by the expert on a haptic fictive probe. The robot "ESTELE" presented in [5] is a light robot mounted on the patient body that was validated for abdominal and fetal echography from clinical trial conducted in 4 medical centers [6]. However, as mentioned in [6], a sonographer paramedic is needed by the side of the patient to hold the robot and to pre-position it on the patient in order to provide to the remote expert a first view of the organ of interest. The expert can then do the examination by teleoperating the probe. Unfortunately, sometimes the expert can lose the anatomical element of interest from the image and in this case the sonographer paramedic should re-position the robot in order to help the expert to retrieve the element of interest. For that reason, a robotic control mode can be useful to automatically maintain the visibility of the section of the element of interest in the US image while the expert tele-operates the probe. A first shared control scheme has been presented in [7] where the 3 degrees-of-freedom (DOF) of the robotized probe outside the US plane (1 translation, 2 rotations) were tele-operated and the 3 DOF lying inside the US plane ( 2 translations, 1 rotation) were controlled by ultrasound visual servoing to center automatically the image on the carotid artery.

In this paper, we propose another approach that gives more flexibility to the expert since all the DOF of the robot holding the probe, both in the US plane and out of the US plane, are shared between the tele-operation and an ultrasound visual task. This latter automatically activates before the observed organ of interest leaves the US observation plane in order to keep it always visible in the image with a minimum section area. This automatic assistance is also useful when the doctor exams an organ that slowly moves due to the physiological motion of the patient since it automatically adapts the probe position in such a way to always keep an intersection of the organ with the US image plane. The structure of this paper is as follows. In Section 2, we briefly present the context of our work and describe the robotic task we propose to perform in order to assist the radiologist. Section 3 presents the multi-task control law we propose. Results obtained from simulation are discussed in Section 4. Finally Section 5 demonstrates the efficiency of the robotic task from experiments performed with a robot actuating a probe in contact with a realistic abdominal phantom.

\section{OBJECTIVE AND DESCRIPTION OF THE ROBOTIC TASK}

Our work is realized within the ANR PROSIT French national project whose goal is to develop an interactive masterslave robotic platform for medical diagnose applications 
with autonomous assistance modes. The "PROSIT" robot presented in [5] which is a modified version of "ESTELE" is shown in Fig.1a. This 4 DOF robot can move the US probe according to: 3 rotations around $\mathrm{X}, \mathrm{Y}$ and $\mathrm{Z}$ axes of the probe frame $\left\{R_{p}\right\}$ and 1 translation along Y-axis of the probe frame. The angular velocities around $\mathrm{X}, \mathrm{Y}$ and $\mathrm{Z}$ axes of $\left\{R_{p}\right\}$ are denoted by $\omega_{x}, \omega_{y}$ and $\omega_{z}$ and $v_{y}$ represents the translation velocity along Y-axis of $\left\{R_{p}\right\}$.

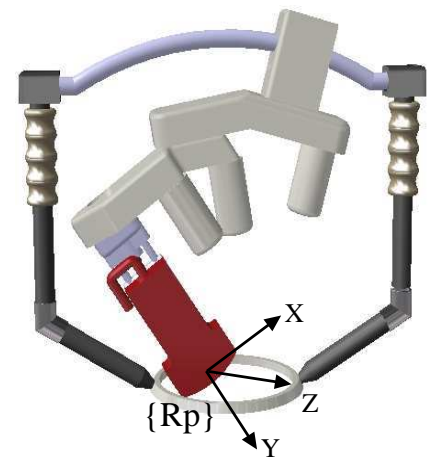

(a)

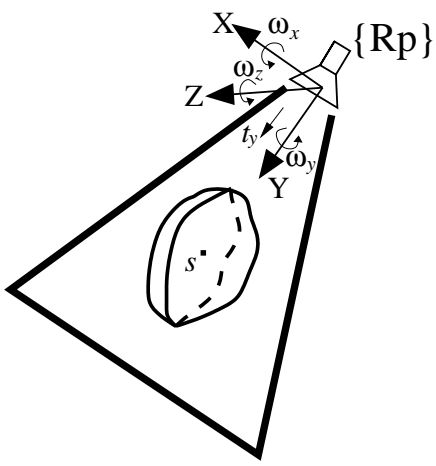

(b)
Fig. 1. (a) The "PROSIT" tele-operable portable 4 DOF robot actuating the US probe. (b) The section $\mathcal{S}$ of the organ of interest observed in the 2D US image.

We describe herein the successive steps of the teleechography procedure aided by our visibility assistance mode:

1) During all the procedure, the robot base is kept by an assistant on the patient body and the current US image is streamed to the expert site via internet or satellite connection.

2) The expert tele-operates all the 4 DOF of the robot with a haptic fictive probe to reach a section of the anatomic object of interest.

3) Once an organ of interest is observed, the expert can decide to activate the "visibility assistance task" by clicking inside a closed organ section $S$ (see Fig.1b). Its $2 \mathrm{D}$ contour $C$ is then automatically extracted from the US image thanks to the real-time active contour algorithm that we presented in [8]. A set of visual features are then computed from $C$ in order to track in real time the evolution of the considered organ section.

4) The doctor can then freely tele-operate all the 4 DOF unless one or several visual features go close to fixed limits. If this case occurs then the system automatically prevents the loss of the organ visibility by acting like an active virtual guide and the doctor can still teleoperate the DOF in the direction that does not degrade the visibility.

5) At any time the doctor can go back to the full teleoperation control mode to examine other organs.

\section{MULTI-TASK CONTROL LAW}

The classical visual servoing methods [9] use images acquired by a camera. In this case, the $2 \mathrm{D}$ image is a projection of the $3 \mathrm{D}$ world so that the coordinates of $2 \mathrm{D}$ visual features are usually used to control the 6 DOF of a robotic system. However, a 2D US probe only provides information in its observed plane. Therefore, suitable features need to be used to control not only the in-plane velocity $\left(\omega_{z}\right)$ but also the out-of-plane velocity $\left(\omega_{x}\right.$ and $\left.\omega_{y}\right)$ of our 4 DOF robotized probe to maintain the indicated section in sight.

We denote $\mathbf{v}=\left(v_{y}, \boldsymbol{\omega}\right)$ the probe velocity expressed in the probe control frame, where $v_{y}$ is the in-plane linear velocity and $\boldsymbol{\omega}$ is the angular velocity. As it is usual in ultrasound visual servo [10], $v_{y}$ is regulated by a force control scheme in order to maintain a constant contact force between the probe and the patient. The visual servoing and the tele-operation regulate the angular motion $\boldsymbol{\omega}$. First, we present how the visibility constraint can be ensured with ultrasound visual servo. We then combine the visibility task with the teleoperation.

\section{A. Ultrasound visibility constraints}

There are several ways an object can leave an ultrasound image. As in classical images, the section of interest $\mathcal{S}$ can of course leave the field of view by passing the image border. Out-of-plane motions can also make the object disappear: in this case, the surface of $\mathcal{S}$ progressively becomes null and the visibility is lost. The set of visual features that handle visibility is thus defined by:

$$
\mathbf{s}=\left(x_{g}, y_{g}, d\right)
$$

where $\left(x_{g}, y_{g}\right)$ are the coordinates of the center of gravity of $\mathcal{S}$ and $d$ is the square root of its surface. We take the square root instead of the actual surface in order to deal with homogeneous coordinates in $\mathbf{s}$.

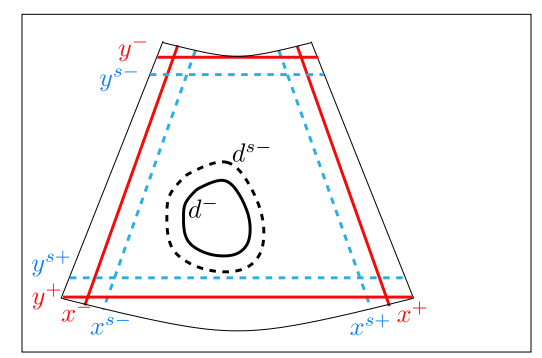

Fig. 2. Visibility constraints. Visibility (plain red) and safe (dotted blue) intervals are defined for $x_{g}$ and $y_{g}$. Minimum (plain black) and safe (dotted black) values are defined for $d$.

Fig. 2 sketches the constraints that we predetermine to keep the section in the field of view. The constraints correspond to the conditions:

$$
\left\{\begin{array}{l}
x_{g} \in\left[x^{-}, x^{+}\right] \\
y_{g} \in\left[y^{-}, y^{+}\right] \\
d \geq d^{-}
\end{array}\right.
$$

where $\left[x^{-}, x^{+}\right]$is the visibility interval for $x_{g},\left[y^{-}, y^{+}\right]$is the visibility interval for $y_{g}$ and $d^{-}$is the minimum value for $d$. As the field of view is rendered by a circular sector, the value of $x^{-}$and $x^{+}$vary in function of $y_{g}$ to adapt its form. 
A classical way to deal with the exposed constraints (2) is to define for each feature a safe interval that triggers the servoing [11]. Similarly to (2), the visual feature safe region is defined as:

$$
\left\{\begin{array}{l}
x_{g} \in\left[x^{s-}, x^{s+}\right] \\
y_{g} \in\left[y^{s-}, y^{s+}\right] \\
d \geq d^{s-}
\end{array}\right.
$$

An example of safe region is represented in Fig.2. We now expose the control law that ensures that the defined constraints are respected.

\section{B. A control law that copes with constraints}

In classical visual servoing schemes, desired values $\mathbf{s}^{*}$ are defined for each visual feature. The corresponding error $\mathbf{e}=$ $\mathbf{s}-\mathbf{s}^{*}$ is then regulated to 0 with the following control law:

$$
\boldsymbol{\omega}=-\lambda \widehat{\mathbf{L}}_{\omega}^{+} \mathbf{e}
$$

where $\lambda$ is the control gain and $\widehat{\mathbf{L}}_{\omega}$ is angular part of the estimated interaction matrix of $\mathbf{s}$ expressed in the control frame [9]. $\widehat{\mathbf{L}}_{\omega}^{+}$denotes the Moore-Penrose pseudo-inverse of $\widehat{\mathbf{L}}_{\omega}$. Such control laws are known to have nice properties in terms of stability and robustness with regards to calibration imprecisions.

In our case, we want to control each visual feature only when its value approaches a constraint. The desired values are chosen in the safe region for all features: $\left(x_{g}^{*}, y_{g}^{*}\right)$ is defined from the center of the image and $d^{*}=d^{s-}$ corresponds to the surface when the visibility task begins. In [11] a weighted error, denoted $\mathbf{H e}$, is defined so as to ensure the visibility constraints in classical visual servoing. $\mathbf{H}$ is a diagonal weighting matrix that allows continuous adding or removing of visual features in the control law [12]. In our approach, $\mathbf{H}=\operatorname{Diag}\left(h_{x}, h_{y}, h_{d}\right)$ where $h_{x}, h_{y}$ and $h_{d}$ are the varying weights that correspond to the visual features from (1). Adapting the weighting formulation from [11] to our visual features yields:

$$
\begin{aligned}
& h_{x}\left(x_{g}\right)= \begin{cases}\frac{x_{g}-x^{s+}}{x^{+}-x_{g}} & \text { if } x_{g}>x^{s+} \\
\frac{x_{g}-x^{s}}{x^{-}-x_{g}} & \text { if } x_{g}<x^{s-} \\
0 & \text { else }\end{cases} \\
& h_{y}\left(y_{g}\right)=\left\{\begin{array}{cc}
\frac{y_{g}-y^{s+}}{y^{+}-y_{g}} & \text { if } y_{g}>y^{s+} \\
\frac{y_{g}-y^{s-}}{y^{-}-y_{g}} & \text { if } y_{g}<y^{s-} \\
0 & \text { else }
\end{array}\right. \\
& h_{d}(d)=\left\{\begin{array}{cc}
\frac{d-d^{s-}}{d^{-}-d} & \text { if } d<d^{s-} \\
0 & \text { else }
\end{array}\right.
\end{aligned}
$$

The corresponding function is represented in Fig.3. The weight is null in the safe interval, and increases to infinite as the feature value approaches its limits. A constraint is said to be activated as soon as its weight is non-null. In [11] the control law that regulates $\mathbf{H e}$ to 0 yields:

$$
\boldsymbol{\omega}=-\lambda\left(\mathbf{H} \widehat{\mathbf{L}}_{\omega}\right)^{+} \mathbf{H e}
$$

A strong hypothesis to use this control law is that $\mathbf{H} \widehat{\mathbf{L}}_{\omega}$ should be always full rank. This is not the case in our

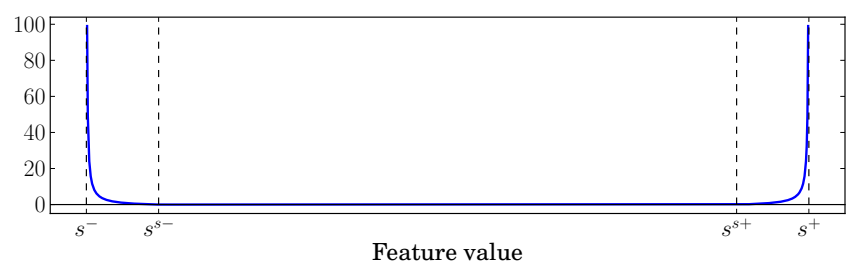

Fig. 3. Weighing function. $h$ is null in the safe interval and increases to the border.

scheme, as $\mathbf{H} \widehat{\mathbf{L}}_{\omega}$ can be full rank 3 if all features are in the unsafe area, but may also be null if all features are in the safe region as it implies that $\mathbf{H}=0$. As we will show in simulation, using (8) leads to a discontinuous control law. The framework of varying-feature-set [12] proposes a new pseudo-inverse operator, that allows using a varying-rank weighting matrix $\mathbf{H}$. However, the formulation is appropriate for weights between 0 and 1 . As $\mathbf{H}$ is defined up to a scale factor, we define the normalized weighting matrix $\mathbf{H}_{n}$ as:

$$
\mathbf{H}_{n}= \begin{cases}\mathbf{H} & \text { if }\|\mathbf{H}\|_{\infty} \leq 1 \\ \mathbf{H} /\|\mathbf{H}\|_{\infty} & \text { else }\end{cases}
$$

In the sequel, we denote $\mathbf{L}_{\omega}^{\oplus} \mathbf{H}_{n}$ the continuous inverse of $\mathbf{L}_{\omega}$ with the weighting matrix $\mathbf{H}_{n}$ (see [12] for the analytical computation). Finally, the control law that ensures the visibility constraints yields:

$$
\boldsymbol{\omega}=-\lambda \mathbf{L}_{\omega}^{\oplus} \mathbf{H}_{n} \mathbf{e}
$$

This control law induces no motion when all features are in their safe region. When a feature approaches a limit, the induced motion tries to make it go back to the safe region. We now explicit the interaction matrix that is used in the control law.

\section{Modeling of the interaction matrix}

The global interaction matrix of the US features can be written $\mathbf{L}_{s}=\left[\begin{array}{ll}\mathbf{L}_{v} & \mathbf{L}_{\omega}\end{array}\right] \in \mathbb{R}^{6 \times 6}$ where $\mathbf{L}_{v}$ is the linear part and $\mathbf{L}_{\omega}$ is the angular part that is actually used for the proposed control. Denoting ${ }^{s} \mathbf{T}_{p}$ the twist transformation matrix that transforms velocity skews from the probe frame to the image center frame, $\mathbf{L}_{s}$ yields:

$$
\mathbf{L}_{s}=\widetilde{\mathbf{L}}_{s}{ }^{s} \mathbf{T}_{p}
$$

where $\widetilde{\mathbf{L}}_{s}$ denotes the interaction matrix of the US features expressed in the image center. The three visual features $x_{g}$, $y_{g}$ and $d$ of the object section can be computed from the image moments of a segmented US section as follows:

$$
\left\{\begin{array}{l}
x_{g}=\frac{m_{10}}{m_{00}} \\
y_{g}=\frac{m_{01}}{m_{00}} \\
d=\sqrt{m_{00}}
\end{array}\right.
$$

where $m_{i j}$ is the image moments of the current section $\mathcal{S}$ defined by: $m_{i j}=\iint_{\mathcal{S}} x^{i} y^{j} d x d y$. The time variation of $\mathbf{s}$ can thus be expressed as follows:

$$
\dot{\mathbf{s}}=\left\{\begin{array}{l}
\dot{x}_{g}=\frac{m_{00}}{m_{00}^{2}} \dot{m}_{10}-\frac{m_{10}}{m_{00}^{2}} \dot{m}_{00} \\
\dot{y}_{g}=\frac{m_{00}}{m_{00}^{2}} \dot{m}_{01}-\frac{m_{01}}{m_{00}^{2}} \dot{m}_{00} \\
\dot{d}=\frac{1}{2 \sqrt{m_{00}}} \dot{m}_{00}
\end{array}\right.
$$


The interaction matrix $\widetilde{\mathbf{L}}_{i j}$ related to the image moment $m_{i j}$ is given in [13] for the case of a US image section:

$$
\widetilde{\mathbf{L}}_{i j}=\left[\begin{array}{llllll}
m_{v x} & m_{v y} & m_{v z} & m_{\omega x} & m_{\omega y} & m_{\omega z}
\end{array}\right]
$$

with:

$$
\left\{\begin{array}{l}
m_{v x}=-i m_{i-1, j} \\
m_{v y}=-j m_{i, j-1} \\
m_{v z}={ }^{x} m_{i, j}-{ }^{y} m_{i, j} \\
m_{\omega x}={ }^{x} m_{i, j+1}-{ }^{y} m_{i, j+1} \\
m_{\omega y}=-{ }^{x} m_{i+1, j}+{ }^{y} m_{i+1, j} \\
m_{\omega z}=i m_{i-1, j+1}-j m_{i+1, j-1}
\end{array}\right.
$$

where the terms ${ }^{x} m_{i, j}$ and ${ }^{y} m_{i, j}$ are computed as follows:

$$
\left\{\begin{array}{l}
{ }^{x} m_{i, j}=\oint_{S} x^{i} y^{j} K_{y} d x \\
{ }^{y} m_{i, j}=\oint_{S} x^{i} y^{j} K_{x} d y
\end{array}\right.
$$

The coefficients $K_{y}$ and $K_{x}$ necessitate the knowledge of the normal vector ${ }^{s} \nabla \mathbf{F}$ of the object local surface at each contour point delimiting the observed section. It is used to express the moments variation due to the out-of-plane motions of the probe. We on-line estimate this vector like in [13] by considering successive images with their relative 3D positions obtained from the robot odometry. From (15) and (13), we can express the interaction matrix $\widetilde{\mathbf{L}}_{s}$ of the considered visual features $\mathbf{s}$, expressed in the image center:

$$
\widetilde{\mathbf{L}}_{s}=\left[\begin{array}{c}
\frac{m_{00}}{m_{00}^{20}} \widetilde{\mathbf{L}}_{10}-\frac{m_{10}}{m_{00}^{2}} \widetilde{\mathbf{L}}_{00} \\
\frac{m_{00}}{m_{00}^{2}} \widetilde{\mathbf{L}}_{01}-\frac{m_{00}}{m_{00}^{2}} \widetilde{\mathbf{L}}_{00} \\
\frac{1}{2 \sqrt{m_{00}}} \widetilde{\mathbf{L}}_{00}
\end{array}\right]
$$

From (11) and (17), the interaction matrix $\mathbf{L}_{\omega}$ that is used in the control law is defined. We now expose how the visibility constraints are handled during the tele-operation.

\section{Fusing visibility with the haptic probe}

We denote $\boldsymbol{\omega}_{m}=\left(\omega_{x m}, \omega_{y m}, \omega_{z m}\right)$ the angular velocities that are measured from the haptic probe that is manipulated by the expert. The goal of the control law is to ensure that the actual probe velocity $\boldsymbol{\omega}$ is the nearest possible to the tele-operated value $\boldsymbol{\omega}_{m}$ under the constraint of visibility.

The redundancy framework allows such hierarchical approach for the classical pseudo-inverse control law (8). In this case, the global law yields:

$$
\boldsymbol{\omega}=-\lambda\left(\mathbf{H} \widehat{\mathbf{L}}_{\omega}\right)^{+} \mathbf{H e}+\mathbf{P} \boldsymbol{\omega}_{m}
$$

where $\mathbf{P}=\mathbb{I}_{3}-\left(\mathbf{H} \widehat{\mathbf{L}}_{\omega}\right)^{+}\left(\mathbf{H} \widehat{\mathbf{L}}_{\omega}\right)$ is a projection operator on the null space of the visibility task. However, in this case some of the components of $\boldsymbol{\omega}_{m}$ are totally ignored as soon as one constraint is activated, even partially. Furthermore, the behavior is discontinuous when weights are activated or deactivated. A more general operator $\mathbf{P}_{\oplus \mathbf{H}_{n}}$ has been proposed in [14] for the varying-feature-set framework [12]:

$$
\mathbf{P}_{\oplus \mathbf{H}_{n}}=\mathbb{I}_{3}-\mathbf{L}_{\omega}^{\oplus \mathbf{H}_{n}} \mathbf{L}_{\omega}
$$

Such an operator allows to partially project the components of $\boldsymbol{\omega}_{m}$ when the normalized weighting matrix $\mathbf{H}_{n}$ has intermediary weights. The tele-operated values $\boldsymbol{\omega}_{m}$ are thus

reduced, but not ignored, when the weights are non-null. The corresponding control law yields:

$$
\boldsymbol{\omega}=-\lambda \mathbf{L}_{\omega}^{\oplus} \mathbf{H}_{n} \mathbf{e}+\mathbf{P}_{\oplus \mathbf{H}_{n}} \boldsymbol{\omega}_{m}
$$

Such a control law has been used only in highly-redundant systems [14] that deal with a limited number of constraints. In our approach, the formulation of the weights allows to ensure 3 constraints while performing a tele-operated task of dimension 3, on a probe that has only 3 DOF. In the next section, simulations allow comparing control laws (18) and (20).

\section{SimUlation VALIDATION}

The simulator we have developed displays a binary volume which contains a sphere (Fig.4a) to simulate the organ of interest and generates the binary images observed by a controllable 2D virtual probe. An example of binary image is shown in Fig.4b.

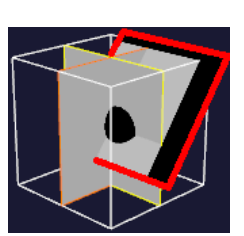

(a) Volume loaded in the simulator and age the virtual probe plane (displayed in red)

Fig. 4. Simulation environment. 3D volume (a), 2D image (b) and operated velocity (c). The generated image observed by the virtual probe where the section of interest is segmented by the use of an active contour (displayed in red).

Red and blue lines indicate the visibility and the safe intervals. The object center of gravity is extracted and displayed in red. We now compare control laws (18) and (20) with the same predefined velocities.

The desired value for the features has been set at the image center $\left(x_{g}^{*}, y_{g}^{*}\right)=0 m$ and with a minimum area corresponding to $d^{*}=0.016 \mathrm{~m}$. Fig.5a and $5 \mathrm{~b}$ represent the evolution of the features during the servoing. They are normalized in order to compare easily their position in the visibility region (corresponding to $[-1,+1]$ ) and the safe region (plotted in dotted lines). In both cases, the features stay in the visibility region. $x_{g}$ (red) quickly reaches the upper safe value $x^{s+}$, while $d$ (blue) reaches several times its lower safe value $d^{s-} . y_{g}$ (green) oscillates in the safe region. Oscillations appear in the discontinuous control law (Fig.5a). The corresponding weights are represented in Fig.5c and 5d. Although the weighting function may increase to infinite, small values are enough to ensure the constraints. The activation clearly corresponds to the configuration where features $x_{g}$ and $d$ are near to their limits. The weighting is very different from one scheme to another, revealing very small weights for the discontinuous control law (Fig.5c). Finally, Fig.5e and 5f represent the actual probe velocity. Very high oscillations are observed with the discontinuous 


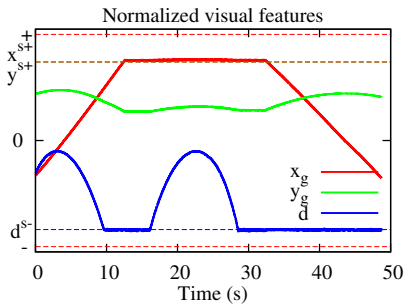

(a) Features with law (18)

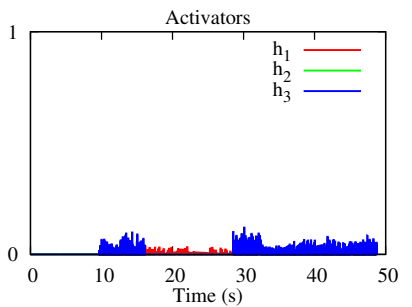

(c) Weighting of law (18)

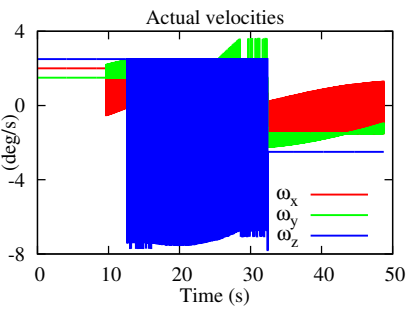

(e) Velocity with law (18)

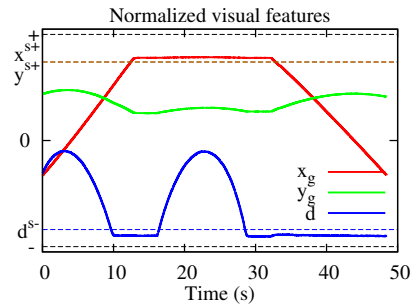

(b) Features with law (20)

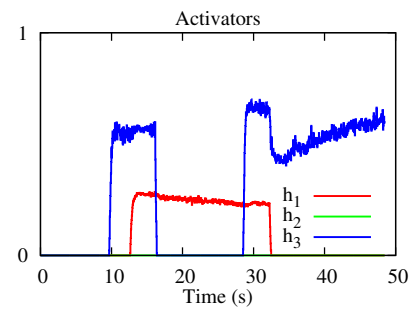

(d) Weighting of law (20)

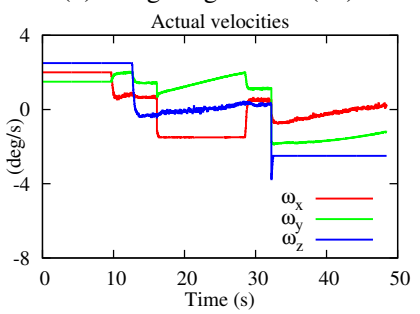

(f) Velocity with law (20)

Fig. 5. Simulation results for control laws (18) (left) and (20) (right) Normalized visual features (top), weight values (middle) and actual probe velocity (bottom).

control law (Fig.5e). On the opposite, our scheme (Fig.5f) follows at best the predefined velocity from Fig.4c. Changes only appear when some features are near to their limits, that is when the visibility constraints are activated.

\section{ROBOTIC EXPERIMENTS}

Now we present results obtained from real robotic experiments. The experimental setup is shown in Fig.6a. An anthropomorphic 6 DOF robot arm equipped with a force sensor holds a convex 2-5 MHz US transducer that interacts with a US abdominal phantom. We purposely constraint the motion of the robot in order to simulate the 4 DOF cinematic of the "PROSIT" robot. That means that only the velocity vector $\mathbf{v}=\left(v_{y}, \omega_{x}, \omega_{y}, \omega_{z}\right)$ can be applied to the probe. A realistic abdominal phantom simulates the patient body and the automatic segmentation of the organ of interest is realtime performed ( 25 frames/s) by our efficient active contour algorithm that we presented in [8]. In the following robotic experiments, $v_{y}$ is regulated by the force control scheme implemented in [10] in order to maintain a $1 N$ contact force between the probe and the phantom. Similar desired values are defined: $\left(x_{g}^{*}, y_{g}^{*}\right)=0 m$ and $d^{*}=0.021 m$. The main task gain is $\lambda=0.4$.

First we move the phantom by hand (see the video accompanying this paper). No velocity is tele-operated and the goal is to ensure visibility during the phantom motion. The robot moves accordingly to the phantom motion. Fig.6b briefly represents the feature weights (top) and the US image

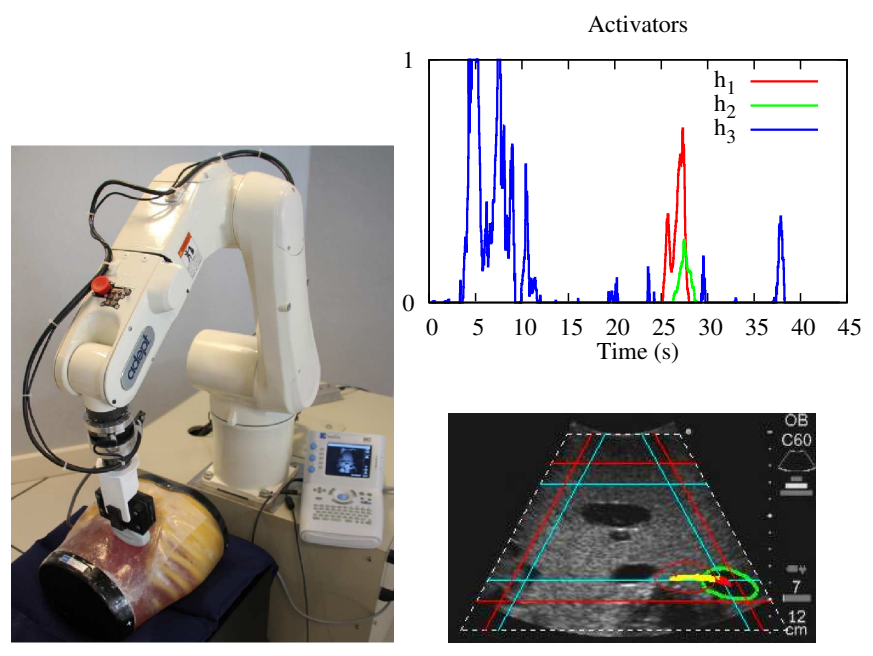

(a) Viper robot with abdominal phantom.

(b) Visibility is ensured during motion.

Fig. 6. The experimental setup is composed by a robot holding a US probe that interacts with a realistic abdominal phantom (a). During phantom motion, the constraints are activated and the visibility is ensured (b).

(bottom): constraints are activated and the object stays in the safe region. We now detail the results when tele-operated velocities are sent to the probe. The operated velocities are represented in Fig.7b. Without control for the visibility, the surface of interest leaves the image as seen in Fig.7a. The trajectory of the center of gravity is displayed in yellow when no constraints are taken into account and in red when at least one constraint is activated. It is displayed in white when the feature leaves the visibility region. White parts do appear in Fig.7a at both extremities, revealing that $x_{g}$ leaves the acceptable interval. Furthermore, the object area is particularly small in Fig.7a. On the opposite, Fig.7d shows that active control prevents the object from leaving the image: the trajectory does not pass the red lines, and the object is larger in the image.

Three red parts are visible: on both sides of the image, which means $x_{g}$ was leaving its safe interval, and in the middle of the image, that corresponds to the activation of $y_{g}$. This observation is confirmed by Fig.7e. The evolution of $x_{g}$ (red) shows that it first approaches its upper limit $(t=30 \mathrm{~s})$, then approaches its lower limit $(t=45 s)$. This corresponds to the extremities of the trajectory. The upper limit of $y_{g}$ (green) is approached many times, the feature value is indeed very near to the upper safe value. Finally, the area of the surface of interest (blue) is controlled several times when approaching its lower limit. The weights are represented in Fig.7f. The activation of each weight correspond to the US visual feature approaching its limit. The object area (blue) is activated particularly often, denoting that the operated velocity $\boldsymbol{\omega}_{m}$ would make the object surface reduce too much. The abscissa $x_{g}$ is activated twice, that corresponds to the two extremities already mentioned. Let us note that a weight of 0.6 is enough to ensure the visibility constraint at $t=25 \mathrm{~s}$. Although it is always near its upper safe value, the ordinate $y_{g}$ has very small weights during the scheme. The probe actual velocity (Fig.7c) are very similar to the tele-operated ones (Fig.7b). This shows that the user can still tele-operate the probe 
Operated velocities

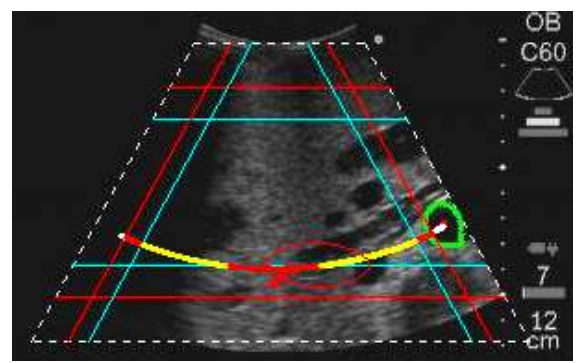

(a) US image without control. $x_{g}$ (white) leaves the visibility area. Surface is small.

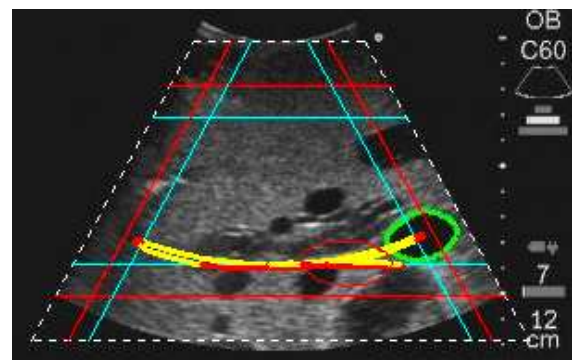

(d) US image with control. $x_{g}$ goes back in the safe area.

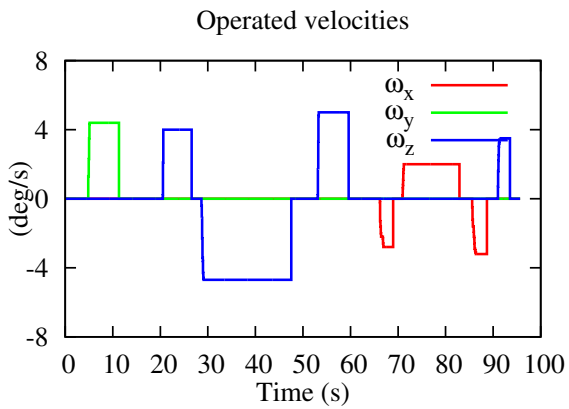

(b) Operated velocity $\boldsymbol{\omega}_{m}$

Normalized visual features

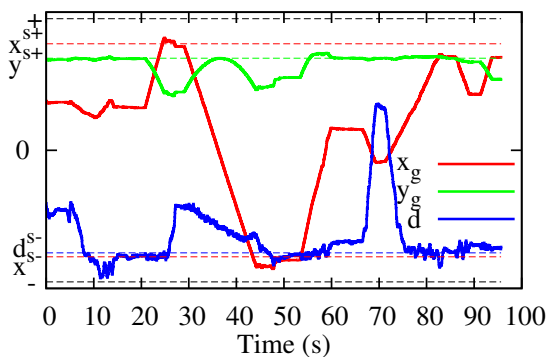

(e) Visual features. The visibility constraint is respected.

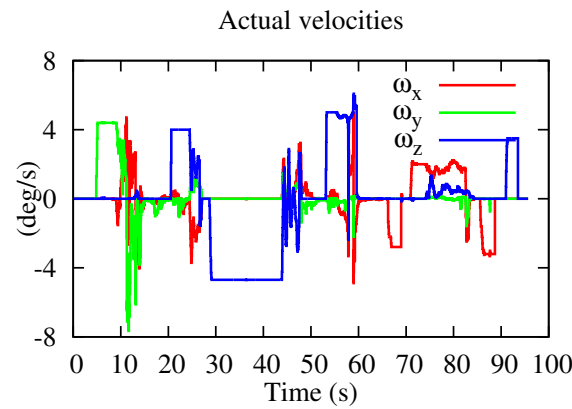

(c) Probe velocity $\boldsymbol{\omega} . \boldsymbol{\omega}$ follows $\boldsymbol{\omega}_{m}$ at best under the visibility constraint.

Activators

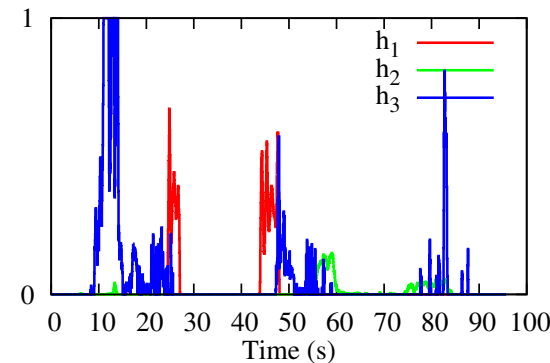

(f) Feature weights. All features are activated during this experiment.

Fig. 7. Results on the real phantom. US image without (a) and with (d) the control law. Operated velocity $\boldsymbol{\omega}_{m}$ (b) and actual probe velocity $\boldsymbol{\omega}$ (c). Normalized visual features (e) and corresponding weights (f).

correctly while the visibility assistance task is active.

\section{CONCLUSION}

We presented an assistance control mode that automatically maintains the visibility of an organ during teleechography examination performed with a tele-operated robot holding a 2D US probe. The main advantage of the proposed multi-task control law is that the DOF of the robotized probe are not exclusively constraint by the visibility task but also available for the tele-operation. Experimental result shows that the expert can explore the organ of interest without caring if it will leave the US view. Future works will concern the implementation of the proposed control system on the "PROSIT" robot and clinical validation during teleechography examinations.

\section{ACKNOWLEDGMENT}

The authors acknowledge the support of the ANR project PROSIT of the French National Research Agency and the S2E2 cluster.

\section{REFERENCES}

[1] W. Chimiak, R. Rainer, N. Wolfman, and W. Covitz, "Architecture for a high-performance tele-ultrasound system," in Medical Imaging: PACS Design and Evaluation: Engineering and Clinical Issues, vol. 2711, 1996, p. 459-465.

[2] E. Degoulange, L. Urbain, P. Caron, S. Boudet, J. Gariepy, J.-L. Megnien, F. Pierrot, and E. Dombre, "Hippocrate: an intrinsically safe robot for medical applications," in IEEE/RSJ Int. Conf. on Intelligent Robots and Systems, IROS'98, vol. 2, October 1998, pp. 959 -964.

[3] K. Masuda, E. Kimura, N. Tateishi, and K. Ishihara, "Three dimensional motion mechanism of ultrasound probe and its application for tele-echography system," in IEEE/RSJ Int. Conf. on Intelligent Robots and Systems, IROS'01, vol. 2, October 2001, pp. 1112 -1116.
[4] A. Vilchis, J. Troccaz, P. Cinquin, K. Masuda, and F. Pellissier, "A new robot architecture for tele-echography," IEEE Trans. Robot. Autom., vol. 19, no. 5, pp. 922 - 926, october 2003.

[5] L. Nouaille, N. Smith-Guerin, G. Poisson, and P. Arbeille, "Optimization of a 4 dof tele-echography robot," in IEEE/RSJ Int. Conf. on Intelligent Robots and Systems, IROS'10, October 2010, pp. 3501 3506 .

[6] P. Arbeille, J. Ayoub, V. Kieffer, P. Ruiz, B. Combes, A. Coitrieux, P. Herve, S. Garnier, B. Leportz, E. Lefbvre, and F. Perrotin, "Realtime tele-operated abdominal and fetal echography in 4 medical centres, from one expert center, using a robotic arm \& isdn or satellite link," in IEEE Int. Conf. on Automation, Quality and Testing, Robotics, AQTR'08, vol. 1, May 2008, pp. $45-46$.

[7] P. Abolmaesumi, S. Salcudean, W.-H. Zhu, M. Sirouspour, and S. DiMaio, "Image-guided control of a robot for medical ultrasound," IEEE Trans. Robot. Autom., vol. 18, no. 1, pp. 11 -23, February 2002.

[8] T. Li, A. Krupa, and C. Collewet, "A robust parametric active contour based on fourier descriptors," in IEEE Int. Conf. on Image Processing, ICIP'11, Brussels, Belgium, September 2011.

[9] F. Chaumette and S. Hutchinson, "Visual servo control, part i: Basic approaches," IEEE Robot. Autom. Mag., vol. 13, no. 4, pp. 82-90, December 2006.

[10] C. Nadeau and A. Krupa, "Intensity-based direct visual servoing of an ultrasound probe," in IEEE Int. Conf. on Robotics and Automation, ICRA'11, May 2011, pp. $5677-5682$.

[11] O. Kermorgant and F. Chaumette, "Combining ibvs and pbvs to ensure the visibility constraint," in IEEE/RSJ Int. Conf. on Intelligent Robots and Systems, IROS'11, San Francisco, USA, September 2011.

[12] N. Mansard, A. Remazeilles, and F. Chaumette, "Continuity of varying-feature-set control laws," IEEE Trans. Autom. Control, vol. 54, no. 11, pp. 2493-2505, November 2009.

[13] R. Mebarki, A. Krupa, and F. Chaumette, "2-d ultrasound probe complete guidance by visual servoing using image moments," IEEE Trans. Robot., vol. 26, no. 2, pp. 296 -306, April 2010.

[14] N. Mansard, O. Khatib, and A. Kheddar, "A unified approach to integrate unilateral constraints in the stack of tasks," IEEE Trans. Robot., vol. 25, no. 3, pp. 670-685, 2009. 